\title{
Perception sémantique et perception sémiotique: propositions pour un modèle perceptif du signe linguistique
}

Régis Missire

Université de Toulouse 2 - Fr regis.missire@univ-tlse2.fr

\begin{abstract}
Résumé: Cet article entend considérer le thème de la perception tel qu'il est développé dans le contexte de la conception morpho-sémantique de l'activité langagière, selon quelle langue, avant d'être conçu en sa capacité à enregistrer une perception pré-linguistique ou à assister à la Les effets de ses régularités grammaticales doivent être problématisés sur la façon dont il a été perçu lui-même, à la fois dans le plan du signifiant communément accepté et dans le plan du sens, qui est de plus petite ampleur. Cette conception emprunte ses formes de problématisation aux modèles perceptifs de la Gestalt, basés sur l'hypothèse de perception sémantique, pour laquelle l'interprétation, comprise comme l'attribution de sens à une séquence linguistique, gagne de la place lorsqu'elle est modélisée comme une activité de perception. élaboration des fondamentaux et des formes sémantiques. Cette hypothèse de perception sémantique repose sur une interprétation renouvelée des travaux en psychologie de la perception et en neuropsychologie (RASTIER: 1991) et consiste essentiellement à suivre, sur le plan du sens, les principes perceptifs que nous connaissons qui régissent la perception sensible (cf. par exemple)., opérations d'assimilation dissimilation, principe de bonne continuité, etc.).
\end{abstract}

Mots-clés: Perception sémantique. Perception sémiotique. Modèle perceptuel du signe linguistique.

Resumo: Este artigo pretende considerar o tema percepção tal como foi desenvolvido no âmbito da concepção morfossemântica da atividade da linguagem, segundo a qual, a linguagem, antes de ser concebida em sua capacidade para fazer o registro de uma percepção pré-linguistica ou testemunhar os efeitos de suas regularidades gramaticais, deve ser problematizada sobre o como ela própria tem sido percebida, tanto no plano do significante, comumente admitido, como no plano do significado, que o é em menor escala. Tal concepção toma emprestado suas formas de problematização aos modelos perceptivos da Gestalt, fundamentando-se na hipótese da percepção semântica, para a qual a interpretação, compreendida como atribuição de sentido a uma sequência linguística, ganha espaço ao ser modelizada como uma atividade de percepção/ elaboração de fundamentos e formas semânticas. Esta hipótese da percepção semântica se apoia numa interpretação renovada de trabalhos em psicologia da percepção e em neuropsicologia (RASTIER: 1991) e consiste essencialmente em acompanhar, sobre o plano do significado, os princípios perceptivos que sabemos que regem a percepção sensível (cf por exemplo, as operações de dissimilação de assimilação, o principio da boa continuidade, etc).

Palavras chave: Percepção semântica. Percepção semiótica. Modelo perceptivo do signo linguístico.

\section{Perception sémantique et perception sémiotique : problématique}

Nous envisagerons le thème perceptif tel qu'il a été développé dans le cadre de la conception morphosémantique de l'activité langagière, pour laquelle le langage, avant que d'être envisagé dans sa capacité à formuler le compte rendu d'une perception antélinguistique, ou à en témoigner des effets dans ses régularités grammaticales, doit être problématisé en tant qu'il a lui-même à être perçu, sur les plans du signifiant, ce qui est communément admis, comme du signifié, ce qui l'est moins. Une telle conception emprunte ses manières de problématisation aux modèles perceptifs de la Gestalt, et fait

\footnotetext{
${ }^{1}$ Publicado na revista Texto ! Volume XVIII - $n^{\circ} 2$ (2013). Revista sob licença Creative commons que permite a reprodução, simplesmente citando a fonte.
} 
fond sur l'hypothèse de la perception sémantique, pour laquelle l'interprétation, comprise comme assignation de sens à une suite linguistique, gagne à être modélisée comme une activité de perception/élaboration de fonds et de formes sémantiques. Cette hypothèse de la perception sémantique s'étaye sur une interprétation renouvelée de travaux en psychologie de la perception et en neurophysiologie (Rastier 1991), et consiste pour l'essentiel à reconduire sur le plan du signifié les principes perceptifs que l'on sait régir la perception sensible (cf. par exemple des opérations de dissimilation, d'assimilation, le principe de bonne continuation, etc.). De ce point de vue, il importe de souligner que les travaux menés dans ce cadre problématique depuis une vingtaine d'années visaient moins au développement d'une « nouvelle » théorie sémantique qu'à celui d'un domaine de modélisation pour une théorie sémantique déjà existante, dont ont peut considérer que la sémantique interprétative de F. Rastier, synthèse des sémantiques structurales continentales, est un équivalent assez juste. En ce sens, le modèle morphosémantique permet de convoquer dans une visée heuristique les ressources conceptuelles des théories perceptives, le principe général consistant à établir une équivalence entre concepts de la théorie source (i.e une théorie sémantique) et de la théorie cible (i.e une théorie perceptive qui joue comme domaine de modélisation) de manière à faire ensuite travailler les propriétés et qualités spécifiques de la théorie cible. Par exemple, en proposant de définir un fond sémantique comme un faisceau d'isotopies, et une forme sémantique comme un groupement structuré de sèmes, on assure cette traduction minimale, les spécificités des relations fonds/formes connues par ailleurs, en l'occurrence découvertes dans les études sur la perception, permettant ensuite éventuellement de mieux comprendre la relation entre sème et isotopie : la valeur heuristique de l'analogie initiale consiste ainsi précisément dans le surcroît qualitatif apporté par le domaine de modélisation convoqué. A la croisée de la sémantique structurale et des recherches cognitives intégrant le thème perceptif, un tel cadre problématique a montré des capacités descriptives éclairantes, notamment pour approcher les phénomènes de la textualité du point de vue de l'activité interprétative. Sans prétendre à l'exhaustivité, on mentionnera en particulier l'usage qui a été fait de l'idée fondamentale de transposabilité avec les concepts de métamorphisme et de méréomorphisme pour retravailler les médiations entre les différents niveaux de l'analyse, du morphème au texte, ou encore, dans une perspective plus littéraire, la 
reformulation éclairante des problèmes de thématique et de topique textuelle et intertextuelle (Rastier 2001). Plus récemment, les développements autour du concept de paratopie, ont permis de proposer des descriptions des productions langagières reposant sur des opérations d'amalgame et de décumul sémantique dont la portée paraît généralisable aux productions écrites comme orales (Missire 2005, 2010a, 2011).

Outre le développement de concepts immédiatement descriptifs, la réflexion qui a été menée a également consisté, sur un plan plus théorique, à évaluer les conséquences que le choix d'un tel domaine de modélisation pouvait avoir en retour sur la théorie de sémantique linguistique concernée : de fait, le «surcroît qualitatif » évoqué plus haut devrait en principe pouvoir se relire de façon cohérente dans le corpus théorique initial. Par exemple, s'il est possible de décrire dans un modèle de type morphologique une transition sans solution de continuité des fonds aux formes, et si par ailleurs ce concept paraît éclairant pour rendre compte d'un phénomène sémantique concret, on pourra cependant rencontrer des difficultés à le reformuler dans le cadre théorique initial : l'élargissement profitable des phénomènes décrits apporté par le recours au domaine de modélisation interroge ainsi la théorie en retour. Dans ce contexte, on a pu suggérer que le modèle morphosémantique requiert des évolutions conceptuelles au sein de la sémantique interprétative, dont on a trouvé des modalités intéressantes dans certains aspects de l'énergétisme coserien d'une part, et les propositions pour une théorie des formes sémantiques (Cadiot,Visetti, 2001 ; Visetti, Cadiot 2006) d'autre part (Missire, 2005).

Les réflexions contenues dans cet article se situent sur le même plan des relations entre théorie linguistique et domaine de modélisation, et portent plus particulièrement sur les relations qu'un tel modèle perceptif de l'activité langagière entretient, ou pourrait entretenir, avec certaines des dualités conceptuelles les plus reçues en linguistique (langue/parole; signifié/signifiant; syntagmatique/paradigmatique, etc.), et plus largement avec le concept de signe. Il faut souligner en effet que les concepts descriptifs proposés dans le cadre des travaux sur la perception sémantique ont somme toute été élaborés de manière relativement indépendante des concepts précités, ce qui au final laisse pendantes des questions importantes, par exemple: comment comprendre cette élaboration/perception de fonds et de formes sémantiques en laquelle consisterait l'activité de langage en relation avec l'hypothèse régulatrice de la distinction 
langue/parole? Quel statut assigner au principe d'unités bifaces, dès lors que la perception est posée comme d'emblée sémantique? Comment penser la relation entre des grandeurs dont la manifestation se déploie sur des fenêtres temporelles larges avec l'intuition que les unités, si on les comprend comme des gestalten, devraient pourtant se manifester dans des formats permettant la mémorisation ? En d'autres termes, on souhaite ici évaluer la façon dont peuvent se concilier thème perceptif et thème sémiotique dès lors que l'on n'observe pas en l'état de relation nécessaires immédiates entre ces deux plans de contextualisation et de problématisation. Une autre façon peutêtre plus précise de formuler le problème consiste à dire que l'essentiel des avancées dans ce cadre problématique a consisté à transposer les principes généraux d'organisation du champ perceptif immédiatement sur le plan du signifié, sans problématiser en amont les relations entre plans du signifié et du signifiant, autrement dit sans que la question de la sémiose soit elle-même posée autrement que comme celle de la rencontre:/corrélation de deux plans acquis indépendamment. Sans doute faut-il immédiatement nuancer ce propos en rappelant que des réponses ont bien été apportées à la question des relations entre plans dans le cadre morphosémantique (problématique de la corrélation des formes sémantiques et des formes expressives (Rastier 2007, Missire 2007), modèle « plat » de l'énonciation reposant sur une conception classique du niveau sémiotique (Rastier 2003) ou encore plus récemment une conception sémioherméneutique du passage (Rastier 2007)), mais ce qui demeure, c'est une forme de cloisonnement premier entre plans : thèmes perceptif et sémiotique sont bien coprésents dans la théorie, mais la sémiose est toujours l'affaire de la rencontre (synchronisation/couplage dans la problématique des formes sémantiques et expressives, conditionnalité sémio-herméneutique dans la problématique du passage) entre deux plans dont la séparation n'est pas réellement problématisée puisqu'elle est à vrai dire constitutive de la sémantique comme discipline. Or il faut bien constater comme un hiatus de ce point de vue, puisque le recours à une inspiration phénoménologique pour modéliser l'activité langagière se trouve pourtant précédée par une séparation principielle issue de la théorie linguistique (i.e. plans du signifiant et du signifié) non théorisée dans le cadre d'une théorie du champ perceptif. Sans doute atteint-on ici une limite de la relation entre théorie linguistique et heuristique perceptiviste, limite que l'on peut d'ailleurs tout à fait considérer comme une pierre 
angulaire pour la constitution d'une sémantique scientifique qui impliquerait à un certain moment de substituer des principes externes, comme celui de la sémioticité, au problème phénoménologique de la constitution des objectivités, notamment sémiotiques. Il paraît toutefois légitime de s'interroger sur la possibilité de prolonger le thème phénoménologique dans la perspective morphosémantique, ce qui pourrait consister à s'affronter directement à la question de la phénoménalité du sémiotique. Si les linguistes ne paraissent pas avoir fait de cette question de la perception sémiotique une priorité de leur agenda, la phénoménologie du langage l'a en revanche à plusieurs reprise thématisé, comme on le constate par exemple dans les deux extraits suivants de La prose du monde de Merleau-Ponty :

\begin{abstract}
«Dans un instant, ce flot de paroles s'annule comme bruit, nous jette en plein à ce qu'il veut dire, et, si nous y répondons par des paroles encore, c'est sans le vouloir : nous ne pensons pas plus aux mots que nous disons ou qu'on nous dit qu'à la main même que nous serrons (...) la parole en exercice ne se contente pas de désigner des pensées comme un numéro, dans la rue, désigne la maison de mon ami Paul mais vraiment se métamorphose en elles comme elles se métamorphosent en lui:« métamorphose par quoi les mots cessent d'être accessibles à nos sens et perdent leur poids, leur bruit, et leurs lignes, leur espace pour devenir pensées. Tel est bien le mystère du langage » (pp. 162- 163, citant J. Paulhan à la fin du passage) « quand quelqu'un (...) a su s'exprimer, les signes sont aussitôt oubliés, seul demeure le sens, et la perfection du langage est bien de passer inaperçue » (p. 16).
\end{abstract}

Le constat qu'on y lit, notamment, c'est que ce « mystère », cette «perfection »du langage réside tout à la fois dans une indissolubilité des plans du signifiant et du signifié («se métamorphose ») et dans une dissymétrie foncière entre eux, le « sens », la « pensée » constituant finalement l'essentiel de ce qui est perçu. Somme toute, et outre une confirmation incidente de l'hypothèse de la perception sémantique, on pourra identifier ici une occurrence d'une loi essentielle de l'organisation du champ perceptif, qui doit se répartir entre figure et fond. Et si l'on conçoit de plonger la question sémiotique dans la problématique perceptive, il paraît en première approximation logique d'admettre que signifiants et signifiés ne peuvent en même temps être au centre du champ : dans l'attitude naturelle la plus fréquente du locuteur, le sens de ce qui est dit occupe en effet le centre de l'attention, quand le murmure du langage est relégué à sa périphérie - et c'est ainsi que l'on peut comprendre la citation précédente de MerleauPonty. En remarquant ce fait simple, on se donne cependant les moyens de corréler un 
principe sémiotique (relation nécessaire entre signifiant et signifié) et un principe perceptif en homologuant le premier à une répartition du champ entre centre et périphérie.Cette connexion de la dissymétrie perceptive et de la problématique sémiotique avait déjà très clairement et précisément été décrite par Husserl dans les Leçons sur la théorie de la signification, en particulier dans la quatrième partie du premier chapitre sur « conscience de son de mot et conscience de signification $»^{2}:$ « Cette conscience de signification se construit sur la conscience du son de mot (...) C'est une liaison aux côtés inégaux: le son de mot et l'objectivité nommée ne peuvent pas, de quelque façon que ce soit, permuter leur places. (...) Le signe d'écriture que nous lisons se tient devant nos yeux sur le papier. Mais ce n'est pas à lui que va, dans la conscience normale de signification du mot (ici de la lecture) notre «intérêt ». Nous jetons la vue dessus ; et pourtant nous ne le percevons pas au sens plus prégnant, bien connu, où nous nous tournons vers un objet en tant qu'il est perçu (...).» (1995, p. 39). Et après avoir présenté un ensemble de distinctions permettant de préciser l'opposition de base figure/fond en fonction de l'intensité avec laquelle l'attention investit le champ ${ }^{3}$, Husserl fait correspondre la perception du signifiant à un remarquer primaire et celle du signifié à une visée thématique située plus haut sur l'échelle attentionnelle, ces deux types de «perceptions » étant simultanées et liées: «La conscience de son de mot a manifestement pour fonction, non pas de retenir le remarquer primaire qui est accompli en elle, mais de le conduire à la conscience de signification qui est stimulée en même temps. [...] Il existe ici précisément une unité phénoménologique particulière entre conscience de son de mot et conscience de signification. » $(1995$, p. 45$) .{ }^{4}$ En sorte que si l'on voulait exprimer cette conjonction des thèmes perceptifs et sémiologiques selon le schématisme usuel ${ }^{5}$ du $C L G$, il conviendrait de dissymétriser les deux termes de la dualité, la symétrie entre les deux plans supposant une thématisation minimale

\footnotetext{
${ }^{2}$ J'ai plaisir à remercier David Piotrowski de m'avoir signalé l'existence de ce texte, ainsi que de m'avoir communiqué avant publication son étude «structuralisme et phénoménologie : le pont du signe », dont la troisième section porte sur des questions proches de celles abordées ici.

${ }^{3}$ Par ordre décroissant sur l'échelle attentionnelle : visée thématique, remarquer primaire, remarquer secondaire, arrière-plan) (cf. Husserl, 1995, pp. 40-44).

${ }^{4}$ Sur la mise en relation de cette analyse de Husserl avec la perspective sémiologique saussurienne, nous suivons totalement la conclusion de Piotrowski : «l'organicité des constituants du signe procède de leur modalisation dans l'unité du champ attentionnel de la conscience ; les actes de l'intention signitive instituent les consciences de son de mot et de signification en tant que telles, dans les positions interdépendantes d'objets d'une visée primaire (perception) et thématique (signification) ; ces positions exposent exhaustivement leurs caractères phénoménologiques respectifs et permettent de rendre compte de l'unité doublement fusionnelle et dissymétrique du signifiant et du signifié. » (2010, p. 27).

${ }^{5}$ Et certes apocryphe.
} 


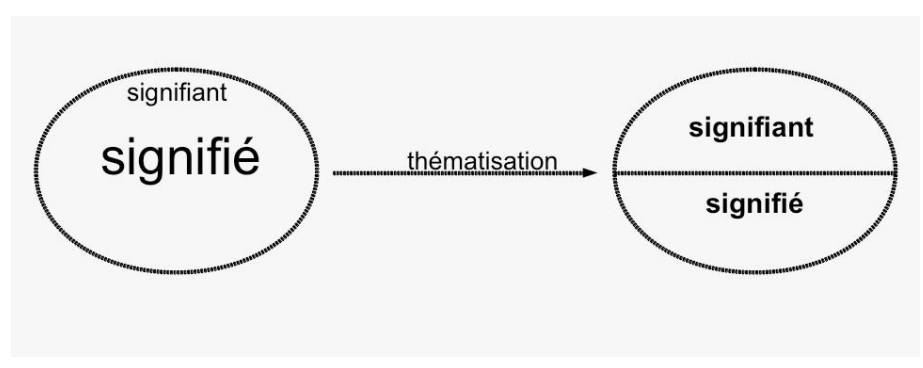

qui peut être aussi bien le fait du linguiste (thématisation métalinguistique) que du locuteur (thématisation épilinguistique) ${ }^{6}$. En d'autres termes, la dualité signifiant/signifié doit faire l'objet d'une compréhension nuancée selon la place qu'on lui assigne sur un axe opposant points de vue méthodologique et réel: du point de vue méthodologique, le partage entre signifiant et signifié est net, et à vrai dire a priori, l'idée sémiologique fondamentale d'une indissociabilité du signifiant et du signifié jouant alors essentiellement comme principe épistémologique administrant les manipulations (commutation, substitution, etc.) auxquelles se livre le linguiste; mais si on déplace sur cet axe le curseur vers la position réelle du locuteur, la distinction signifiant/signifié devient alors beaucoup moins assurée, la même indissociabilité se relisant plutôt dans l'unité phénoménologique de la forme-sens énoncée ou interprétée et la prééminence du plan du signifié dans la conscience. Cette première opposition se voit cependant complexifiée du fait que l'activité linguistique des locuteurs consiste fréquemment à faire varier le curseur, par exemple dans les phases épilinguistiques, reformulantes, où des signifiants sont thématisés comme diversement adéquats (connotation, euphémie/blasphémie, reformulation, etc.) pour une idée à exprimer, ce qui revient en définitive à thématiser la sémiose même, et à donner de fait plus de netteté à l'opposition du signifiant et du signifié. Si la netteté maximale du «vis-à-vis» du signifiant et du signifié est acquise au prix d'une opération de thématisation forte, il faut cependant prévoir, entre cette position d'une part et l'attitude locutoire naturelle ou prévaut le signifié d'autre part, un continuum sur lequel on pourra observer des indifférenciations et des «inversions de phases» entre plans. C'est ce sur quoi nous

\footnotetext{
${ }^{6}$ Il est intéressant de noter que Husserl évoque cette possibilité dans le même chapitre et la même section que celles contenant les réflexions présentées supra: «Il existe ici précisément une unité phénoménologique particulière entre conscience de son de mot et conscience de signification. $C^{\prime} e s t$ sur elle que se fondent ensuite des possibilités essentielles de modification, comme par exemple ces possibilités qui consistent à retourner l'intérêt, à résister à la tendance qui conduit au thème de la signification, ce qui fait que le mot, avec un caractère phénoménologique d'ensemble devenu autre, perd sa signification normale». (1995, p. 46, nous soulignons).
} 
semble insister l'idée d' «étirement» dans le passage suivant de l'étude récente de Rosenthal et Visetti sur la question de l'expression dans une perspective microgénétique, dont l'un des enjeux est précisément de mieux décrire cette profondeur du champ perceptif et la répartition entre ce qu'ils préfèrent appeler expression et contenu: «Le propre de toute sémiose [...] est d'introduire dans l'expérience ce qu'on peut appeler une profondeur, polarisée entre un plan de la «manifestation» et un plan du «contenu». [...] caractère paradoxal de l'expression [...] puisque la profondeur délivrée peut s'étirer jusqu'à se scinder en deux plans dès lors dissociés - l'exprimé $\mathrm{X}$ devenant 'contenu', et l'exprimant Y 'expression'» (2008, pp. 179-187). Reconnaître une telle nécessité d'élaborer un modèle du champ susceptible de connaître des degrés de polarisation entre, notamment, plans sémiotiques, consiste essentiellement à ménager un espace descriptif pour des variations possibles, sans qu'encore le problème des normes propres au domaine envisagé soit posé: outre les thématisations épilinguistiques ou métalinguistiques mentionnées supra il faudrait ainsi évoquer les possibles stylisations, régularités génériques, etc. qui administrent les réquisits attentionnels de chacun des plans ainsi que les modes de signifiance (cf. l'exemple classique de la rime en poésie). De fait, si les formulations empruntées à la phénoménologie du langage que nous venons de présenter nous permettent d'illustrer la nécessité d'étendre la problématique de la perception sémantique à celle d'une perception sémiotique qui l'inclurait, il reste que la généralité tant phénoménologique que sémiotique de ces considérations implique qu'elles soient spécifiées en fonction du domaine d'objectivité concerné, en l'occurrence linguistique. La question qu'il faut donc poser est celle de la possibilité d'objectivation linguistique de ce type de réflexions: plus précisément, peut-on retrouver dans les formes de l'objectivité linguistique et en particulier dans ses unités, certaines des propriétés de champ évoquées, ce qui déterminera in fine le caractère opératoire sur un plan descriptif des préoccupations théoriques précédentes. Parmi les classes d'objet linguistiques sur lesquels nous pouvions faire porter l'analyse, nous avons choisi de nous intéresser au concept d'item lexical de la tradition contextualiste anglo-saxonne, le type de phénomènes auquel il permet d'accéder ayant en effet l'intérêt d'être situé à un palier intermédiaire d'analyse, entre lexème et énoncé, susceptible donc d'être décrits selon ces deux modalités et de faire ainsi communiquer des secteurs souvent trop cloisonnés de la description linguistique. Par ailleurs, les finalités lexicographiques qui 
ont longtemps constitué l'horizon du paradigme théorique au sein duquel le concept d'item lexical a été travaillé sont pour le moins exemptes des préoccupations perceptivistes qui sont les nôtres ici, et il nous a paru significatif que des résultats obtenus dans un tel cadre soient susceptibles de faire l'objet d'une reprise cohérente dans notre perspective. Précisons par ailleurs immédiatement qu'en partant de l'item lexical, nous n'entendons pas y limiter la portée de nos analyses, que nous pensons dans leur principe généralisables au moins à des «entités » proches comme les constructions, collocations, etc ${ }^{7}$ : on s'intéressera ainsi surtout à l'item lexical en tant qu'il paraît pouvoir inspirer un modèle perceptif général du signe linguistique.

\section{L'item lexical comme modèle perceptif du signe}

Élaboré par Sinclair dans une série d'articles ${ }^{8}$ regroupés dans Sinclair (2004), le concept d'item lexical désigne des unités qui ne sont tout à fait ni des lexèmes, ni des syntagmes ou des locutions, mais des configurations complexes qui ne peuvent être déterminées qu'en corpus à partir d'études de cooccurrences. Cette configuration contient, au maximum, un (i) noyau, des (ii) collocations et colligations, (iii) des préférences sémantiques, et (iv) une prosodie sémantique ${ }^{9}$, seuls le noyau et la prosodie étant obligatoires. Le noyau est le point d'entrée pour la recherche d'un item lexical, et Sinclair (2004, pp. 30-34) a par exemple pu montrer par l'analyse exocentrique de l'environnement gauche de naked eye que ce noyau :

- entre en collocation avec the dans $95 \%$ de ses occurrences ;

- entre en colligation avec les prépositions with et to dans $90 \%$ de ses occurrences ;

- entretient une préférence sémantique avec une classe sémantique /visibilité/;

- manifeste une prosodie sémantique de /difficulté/ dans $85 \%$ de ses occurrences :

\footnotetext{
${ }^{7}$ Ces analyses sont d'ailleurs largement inspirées de celles proposées depuis 2006 par Cadiot et Visetti, qui ont progressivement étendu leurs analyses aux constructions, phraséologies et proverbes. ${ }^{8}$ En particulier The search for units of meaning, (1996) et The lexical item (1998).

${ }^{9}$ Introduit par Louw (1993) puis développé parallèlement par Sinclair, le concept de prosodie sémantique (semantic prosody) est défini ainsi par Louw (2000): «a semantic prosody refers to a form of meaning which is established through the proximity of a consistent series of collocates, often characterisable as positive or negative, and whose primary function is the expression of the attitude of its speaker or writer towards some pragmatic situation».
} 


\begin{tabular}{|l|l|l|l|}
\hline \multicolumn{4}{|c|}{ Structure de l'item lexical } \\
\hline prosodie sémantique & préférence sémantique & collocations/colligations & noyau \\
\hline /difficulté/ & /visibilité/ & with, to, the & naked eye \\
difficult, weak, faint, .. & $\begin{array}{l}\text { see, visible, spot, appear, } \\
\text { perceived, apparent, } \\
\text { undetectable }\end{array}$ & & \\
\hline
\end{tabular}

Des énoncés comme too faint to be seen with the naked eye ou it is not really visible to the naked eye manifestent ainsi les quatre composants de l'item naked eye. Les concepts de collocations et de colligations étant bien connus, nous nous intéresserons ici davantage à celui de prosodie sémantique, en en soulignant quatre aspects intéressant notre propos :

(i) Internalisation des prosodies sémantiques par le noyau. C'est un aspect des prosodies sur lequel a notamment insisté Louw (1993), caractérisant un phénomène proche de ce que désigne le concept d'afférence contextuelle (Rastier, 1987). Le principe essentiel en est celui d'une «imprégnation» de certaines unités par d'autres, le noyau ayant en l'occurrence la capacité de rapatrier les valeurs sémantiques des prosodies et préférences. Mais l'essentiel à noter ici est surtout la grande variabilité de saillance perceptive de ces prosodies dans le noyau. Ainsi, si dans un noyau comme traiter de en français la plupart des locuteurs pourront facilement anticiper une prosodie sémantique dépréciative (cf. annexe 1), dans d'autres verbes comme friser ou frôler, cette perceptibilité de la prosodie, pourtant massive à l'examen de corpus, devient semble-t-il plus délicate (cf. annexe 1). On a par exemple pu constater que dans un corpus d'évaluation d'agents fonctionnaires, toutes les occurrences de certaines et parfois apparaissent dans des contextes d'évaluation négative (à tout le moins sont systématiquement corrélées à une réserve de l'évaluateur dans un contexte plus large d'évaluation positive) alors que les occurrences de toutes et toujours sont systématiquement associées à des évaluations positives, ce dont les évaluateurs ont été les premiers surpris (cf. annexe 2). De ce point de vue, le caractère souvent « infra-perceptif » des prosodies sémantiques intéresse directement la théorie des fonds sémantiques, et il faudrait pouvoir corréler les variations de saillance des prosodies à l'opposition forme/fond ${ }^{10}$.

(ii) Un signifiant inassignable, un signifié difficile à gloser. Si les préférences sémantiques n'ont pas de signifiant fixe, puisqu'il s'agit d'une classe sémantique entrant en cooccurrence avec le noyau, les prosodies sont en outre souvent difficiles à localiser dans leur empan de manifestation. Sinclair remarque ainsi à propos des prosodies : «It is not subject to any convention of

${ }^{10}$ Par ailleurs, on retrouve là également une question lancinante en linguistique de corpus, concernant la valeur. sémantique que l'on peut assigner à des coccurrences statistiquement significatives. 
linguistic realization, and so is subject to enormous variation, making it difficult for a human or a computer to find it reliably. It is a subtle element of attitudinal, often pragmatic meaning and there is often no word in the language that can be used as a descriptive label for it». ( 2004, p. 144). En fait, la prosodie sémantique ne trouve pas nécessairement de point de fixation sur une zone de l'item, et ainsi le qualifie intégralement. On l'observe facilement sur le concordancier de parfois dans l'annexe 2: par exemple dans «un léger manque d'empathie peut parfois ternir ses qualités humaines», on identifie bien entendu des lexicalisations de valeurs négatives dans «manque» et «ternir» (d'ailleurs immédiatement euphémisées par «léger» et «peut»), mais au final, l'idée de /réserve de l'évaluateur/ n'appartient exactement à aucun des lexèmes constituant l'item (dont les frontières sont ici particulièrement difficiles à circonscrire dans la mesure où il ne contient qu'un noyau et une prosodie sémantique). Complémentairement à ce caractère inassignable en une zone de l'item, l'explicitation de la valeur sémantique de la prosodie est souvent difficile, et peut justifier le recours à des gloses périphrastiques complexes. Ainsi pour le verbe to budge, Sinclair lexicalise- t-il la prosodie de cette façon: «the user wishes to express or report frustation (or a similar emotion) at the refusal or inability of some obstacle to move, despite pressure being applied» (Sinclair, 2004).

On peut corréler ces deux premiers points en remarquant que plus la prosodie sémantique est aisément perceptible, plus sa lexicalisation sera également simple, susceptible par exemple de trouver une formulation ramassée (p.ex /insulte/ pour «traiter de») et plus sa localisation sera facilement identifiable en un point de la chaîne. En somme, perceptibilité, «localisabilité» et «lexicalisabilité» sont corrélées, leur accroissement faisant passer sans solution de continuité des prosodies aux préférences sémantiques ${ }^{11}$.

(iii) La valeur «pragmatique» des prosodies. Dans les modèles théoriques qui assument la division des tâches entre sémantique et pragmatique, les prosodies sont clairement du côté pragmatique. Pour une perspective qui refuserait un partage aussi net, on dira que les prosodies sont préférentiellement du côté subjectif d'un axe objectif/subjectif, et qu'elles manifestent la position (entendue dans un sens très large) du locuteur par rapport à la situation, et entretiennent donc des affinités avec modalités, évaluation, voire des valeurs illocutoires. A titre d'exemples, outre/difficulté/ pour naked eye, Sinclair dégage la prosodie/impossibilité/ pour true feelings, /menace avec forte charge émotionnelle/pour to brook, ou encore /invitation informelle/ pour my place (dans tous les cas nous simplifions les gloses). Ce caractère subjectif, traduisant la position du locuteur, est d'ailleurs l'une des raisons qui a motivé la dénomination prosodie sémantique.

\footnotetext{
${ }^{11}$ On identifie donc plutôt une différence de degrés entre les deux. Il n'est pas rare d'ailleurs qu'un même terme lexicalise prosodie et préférence: ce serait le cas de «invisible» dans «invisible to the naked eye».
} 
(iv) Le caractère fonctionnel des prosodies sémantiques. Sinclair souligne : « The semantic prosody of an item is the reason why it is chosen, over and above the semantic preferences that also characterize it [...] the whole phrase must be seen as the result of a single choice, with no doubt a number of subsidiary internal choices. The initial choice of semantic prosody is the functional choice which links meaning to purpose ; all subsequent choices within the lexical item relate back to the prosody» (2004; p.32 et p.144, nous soulignons). Ce caractère fonctionnel des prosodies est essentiel, car c'est en partie sur lui que repose l'intuition du caractère unitaire que l'on peut conférer à l'item dans son entier, si l'on convient que la compacité n'est pas le seul critère caractérisant une unité mais qu'il faut également intégrer l'unicité de ce qui motive la profération concrète de cette entité ${ }^{12}$. Dans notre perspective, cette conséquence du principe idiomatique revêt une grande importance, car elle permet de rompre avec une conception probablement trop simple de la représentation de l'énonciation comme une succession monotone de choix paradigmatiques réalisés en différents points de la chaîne syntagmatique, quel que soit par ailleurs l'empan de l'unité choisie (morphème, lexème, locution, etc.). Notamment, cela amène à suggérer que la conception linguistique traditionnelle qui distingue dans les degrés de figement (p. ex. syntagme libre <préférence sémantique <collocation <locution) des grandeurs diversement intégrées en langue peut être complétée en argumentant que ces différents degrés de routinisation participent en réalité de formes unitaires à des degrés variables de sémiotisation, autrement dit des formes dont les différentes «parties » ne sont pas équivalentes dans leurs degrés de corrélation entre plans du signifiant et du signifié. Ceci illustre la variabilité de corrélation et de dominance ${ }^{1213}$ entre signifié et signifiant au sein de l'item lexical : au noyau correspond un signifiant fixe, qui, si on l'isole de ses contextes a la capacité de rapatrier les valeurs environnantes. À partir des préférences sémantiques, c'est au contraire le signifié qui domine, et bien qu'il y ait encore des lexicalisations prototypiques (see et visible sont par exemple des parangons dans le cas de naked eye), seul le trait sémantique /visibilité/ est nécessaire. Quant aux prosodies sémantiques, la valeur manifestée n'est plus nécessairement lexicalisée et devient une qualité globale de la suite linguistique. Sur le schéma suivant, le signe linguistique tel qu'il est généralement entendu se situerait ainsi au degré maximal de corrélation entre signifiant et signifié :

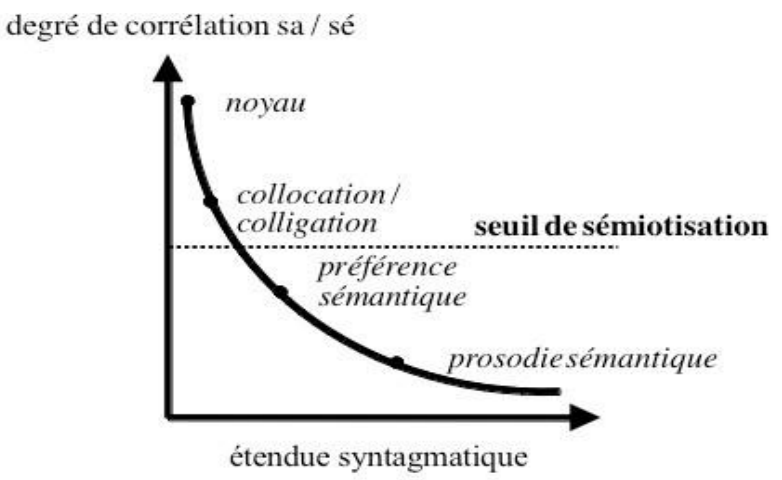

\footnotetext{
12 Martinet ne faisait pas un choix différent en identifiant des monèmes discontinus. Ainsi, dans « /lezanimopes/ les animaux paissent rapproché de /lanimalpè/ l'animal paît, le signe « pluriel » reçoit trois expressions distinctes : /leza.../ au lieu de /la .../, . . mo .../ au lieu de / ... mal .../ et / ... pes/ [...] Il n’y a là, bien entendu, qu'un seul monème de pluriel (...)» (Martinet, 1980, p. 105.).

${ }^{13}$ Nous reprenons cette idée de dominance entre plans à Rastier (2005).
} 
Ainsi, plus l'étendue syntagmatique de manifestation des unités est importante et plus le signifié l'emporte sur le signifiant. Il ne faut pas voir là une forme de conceptualisme en contradiction avec le principe d'unités bifaces: l'affaiblissement du signifiant n'implique pas que les entités soient purement conceptuelles, mais simplement que leur localisation n'est pas toujours strictement déterminable ${ }^{14}$. À l'inverse, la brièveté et la stabilité du noyau lui permettent de fonctionner comme attracteur pour les signifiés des autres parties de l'item lexical : les internalisant, il peut donner l'illusion d'être une unité sa/sé simple.

Si la «fonctionnalité» des prosodies sémantiques doit évidemment être mise en relation avec le principe d'idiomaticité que défend une linguistique comme celle de $\mathrm{J}$. Sinclair, elle peut également être comprise selon d'autres attentes, plus attentives à certaines propriétés phénoménologiques de la parole: si en effet il faut considérer le choix de la prosodie sémantique comme le premier effectué par le locuteur, qui va ensuite en conditionner d'autres «subséquents» au sein de l'item, c'est bien alors le caractère motivationnel de la prosodie qu'il faut reconnaître, son affinité avec un vouloir-dire initial du locuteur qui se concrétisera dans une formulation lui échappant toujours un peu, précisément parce qu'elle est déjà partiellement formulée en langue. On pourrait voir là un corollaire sémiolinguistique de cette remarque de Merleau-Ponty sur l'expérience de la parole: «[...] celui qui parle ou qui écrit est d'abord muet, tendu vers ce qu'il veut signifier, vers ce qu'il va dire, et soudain le flot des mots vient au secours de ce silence, et en donne un équivalent si juste, si capable de rendre à l'écrivain lui-même sa pensée quand il l'aura oublié, qu'il faut croire qu'elle était déjà parlée dans l'envers du monde [...]» (1969, p. 11). En sorte que la variation de dominance entre plans que schématise la figure précédente peut, dans une perspective cette fois énonciative, également se lire comme un balancement sémiotique du signifié

\footnotetext{
${ }^{14}$ Cela apparaîtra peut-être plus clairement si l'on transpose la réflexion à une autre échelle, textuelle par exemple, niveau où l'on peut identifier ce que Bouquet propose de désigner comme signifié suprasegmental pour certains types de valeurs sémantiques qualifiant des textes entiers : "Les valeurs globales du signifié suprasegmental, pour leur part, répondent par définition de l'intégralité de l'unité suprasegmentale considérée, dite texte. On nommera genre - dans un sens technique - ce signifié suprasegmental (...). Ces traits seront susceptibles de consigner des valeurs de nature diverses - appartenant à des catégories usuellement nommées discours, type, mode, champ générique, sous-genre, registre, domaine, niveau, etc. - qui conféreront en outre leur unité aux textes analysés (que ces textes soient une holophrase ou un ouvrage de trois mille pages) (Bouquet, 2007, p. 52). /roman/ est à Madame Bovary ce qu'une prosodie sémantique est à son item lexical.
} 
vers le signifiant, ou plus précisément comme une sémiotisation progressive de ce vouloir-dire dans les formes de la langue, vouloir-dire dont l'énoncé finalement proféré est en quelque sorte le sillage: «Et, bien sûr, pas plus que dans la géométrie le fait physique d'un nouveau tracé n'est une construction, pas davantage dans les arts de la parole l'existence physique des sons, le tracé des lettres sur le papier, ou même la présence de fait de tels mots selon le sens que donne le dictionnaire, de telles phrases toutes faites, ne suffit à faire le sens: l'opération a son dedans et toute la suite des paroles n'en est que le sillage, n'en indique que les points de passage (1969, p. 183, nous soulignons).

On devrait à partir de ces premières réflexions et tentatives de caractérisation commencer de mieux voir en quoi le type de phénoménalité sémiolinguistique auquel permet d'accéder le concept d'item lexical, et alors même qu'il a été élaboré dans un cadre problématique pour le moins éloigné de nos préoccupations, nous permet cependant de formuler des réponses aux interrogations initiales sur les modalités de conciliation des thèmes perceptifs et sémiotiques, notamment relativement à la question du format des unités. Bien entendu, on pourrait se contenter de considérer les noyaux comme des unités, et affirmer, dans une perspective contextualiste en définitive très classique ${ }^{15}$ qu'il faut étudier leur environnement collocationnel pour mieux connaître leur identité. Mais en s'arrêtant là, on reconduirait alors une sorte de modèle concaténatoire qui revient à envisager les productions langagières sur le modèle d'un «collier de signes», quel que soit par ailleurs l'extension syntagmatique que l'on reconnaît au concept de signe. On comprend donc l'intérêt que l'on peut avoir ici à suivre Sinclair quand il passe du constat de la fréquence et de la force des cooccurrences entre mots, classes de mots, etc. à la conclusion que dans la recherche des unités de signification «we must widen our horizons and expect the units of meaning to be much more extensive and varied than is seen in a single word» (2004, p. 39 , nous soulignons). De notre point de vue, cela amène à renverser totalement la manière de formuler le problème habituel: plutôt que partir du signe dyadique déjà donné, symétrique, etc. que l'on cherchera ensuite à situer par rapport aux oppositions régulatrices (langue/parole; paradigmatique/syntagmatique, etc.), on considérera celles-ci (auxquelles on ajoutera donc un axe signifiant/signifié puisqu'on soutient que

${ }^{15} \mathrm{cf}$. le fameux you shall know a word by the company it keeps firthien. 
lui aussi est à conquérir) comme des axes de déploiement d'un champ immédiatement perceptif et sémiotique au sein duquel on identifiera des effets d'unités ainsi que leurs conditions éventuelles d'apparition. L'essentiel est ainsi qu'en s'autorisant à moduler sur les dimensions précédentes, on peut décrire des unités à la méréologie complexe qui font mieux voir la profondeur du champ, l'item lexical apparaissant in fine comme une sorte de dépliage sémiotique partiel. A charge d'approfondissements, on peut déjà formule les remarques suivantes sur les axes de «discernement» usuels:

(i) Axe signifiant/signifié. Le principe de sémioticité (relation nécessaire entre les deux faces du signe) doit être précisé, ce qui implique en l'occurrence de reconnaître une défectivité partielle de l'unité, variable selon que l'on considère la zone des préférences sémantiques ou des prosodies. La variation s'effectue entre 1. un point focal du champ occupé par le noyau dont la qualité de gestalt est garante d'une sémioticité maximale; 2. une zone intermédiaire (préférence sémantique) dont l'empan de manifestation est cependant relativement limité, et dont la sémioticité est plus faible dès lors que la manifestante de la classe sémantique est variable; 3. une sorte de halo (prosodie sémantique) entourant toute la zone, avec un très faible degré de sémiotisation, au point que l'on pourra d'ailleurs simplement parler de fond sémantique, glissant ainsi progressivement d'une caractérisation sémiotique pour 1 . et 2. à une caractérisation essentiellement sémantique pour 3 . Ce faisant, et relativement à la question initiale d'une perception trop immédiatement sémantique et notre souci de lui adjoindre en amont la problématique d'une perception sémiotique à partir de laquelle il serait possible de dériver la première, on voit qu'une telle présentation des choses permet de mettre en relation sans solution de continuité ces deux perceptions, puisque pour une même unité on est amené à reconnaître la prévalence de l'une ou de l'autre $^{16}$. On notera par ailleurs que le niveau où l'on rencontre des unités de format conparable aux items lexicaux (constructions, etc.) est bien de ce point de vue un palier intermédiaire entre un niveau inférieur où se situent des unités plus compactes (morphèmes, lexèmes) avec une sémioticité «stable » et un niveau supérieur (la période et son au-delà) où s'observe au contraire une «défectivité généralisé ${ }^{17}$ : du premier elle conserve la brièveté permettant la mémorisation $^{18}$ et rendant psychologiquement crédible les phénomènes d'afférence ou d'imprégnation; avec le second, outre donc une certaine défectivité, elle partage les relations paratopique et isotopique caractéristiques.

\footnotetext{
${ }^{16}$ On voit cependant qu'il n'y a désormais guère d'intérêt à maintenir cette opposition perception sémantique/perception sémiotique dès lors que la seconde implique la première.

17 Par « défectivité généralisée », on caractérise des unités dont toutes les manifestantes sont variables : ainsi des cooccurrences entre sèmes définissent des formes sémantiques s'appréciant par extraction d'invariants dans des formes de "synonymie élargie », qui peuvent avoir une valeur phraséologique (dépasser les bornes, franchir les limites) ou non (le livre s'articule en trois parties, l'ouvrage s'ordonne en trois sections, etc. Sur ces phénomènes de « coocurrence de sens », cf. notamment Siepman, 2006.

${ }^{18}$ Mémorisation plus manifeste cependant dans des expressions formulaires de type proverbial, qui manifeste d'ailleurs des phénomènes proches de ceux que l'on décrit ici (cf. Visetti, Cadiot, 2006).
} 
De ce point de vue, les types de phénomènes étudiés ici apparaissent comme un «chaînon manquant» particulièrement digne d'intérêt pour une sémantique soucieuse de proposer des modèles unifiés des fonctionnements lexicaux et textuels.

(ii) Axe compact/diffus. Sur cette question, et dans le prolongement de la remarque précédente, on reprendra à notre compte les observations suivantes de V. Rosenthal et Y.-M Visetti: «En raison du métabolisme constant entre formes compactes et formes étales (elle-même perçues de façon plus ou moins holiste), une unité (par exemple un lexème, à un certain niveau de son sémantisme) s'identifiera naturellement à un réseau de formes englobantes et/ou solidaires (collocations, colligations, phraséologies, idiomatismes) dont elle devient du même coup l'index essentiel, et le foyer de coalescence. [...] la forme locale n'est jamais qu'un point de condensation ou de fixation dans un parcours dont les résultats ne s'attestent qu'au niveau de formes et de champs plus englobants» $(2008$, p. 224). Sans poser donc d'alternative entre formats compact et étendu, on constatera plutôt dans les entités concrètes un redoublement entre une zone intense et une zone extense, une sorte d'opposition participative entre un noyau qui d'un côté attire puis sédimente des valeurs environnantes et les autres composants de l'item de l'autre. Ce phénomène, tout à fait général et dépassant largement le cas des unités de type «item lexical», témoigne de la généralité des relations d'isotopie et de paratopie à tous les paliers d'analyse, ainsi que de leur complémentarité (Missire, 2010). Il faut alors insister sur le fait que privilégier l'étude de formes plus compactes et mémorisables comme le sont les noyaux (dans une perspective lexicologique standard) ou bien l'étude de versions plus différenciées et analytiques comparables aux items lexicaux (il ne s'agit bien entendu pas de se limiter à la cartographie proposée par Sinclair et on pourrait tout à fait l'étendre à l'étude des constructions) n'est pas une alternative face à laquelle il faudrait choisir en fonction de préférences disciplinaires ou autres, car l'objectif est bien plutôt de rendre compte et de décrire la co-présence concrète de ces versions amalgamées et décumulées des formes sémiotiques.

(iii) Axe paradigmatique/syntagmatique. La lecture de cette opposition dans le cadre précédent ne semble pas poser de problème ici puisque sa manière de jouer est semblable à celle que l'on retrouve dans des phénomènes bien connus comme les restrictions de sélection. On retiendra donc surtout que l'axe paradigmatique ne doit pas seulement être envisagé comme une dimension externe sur laquelle se disposent des unités entre lesquelles le locuteur aurait le choix à un moment donné de l'énonciation, ce qu'il est évidemment aussi, mais également comme un axe de variation interne à l'unité en certaines de ses localités (cf. les variations de lexicalisation des préférences sémantiques). S 'agissant par ailleurs de la syntagmatique interne de l'unité, on mentionnera, à charge d'approfondissements cependant pour le français, le constat de Sinclair que le noyau est fréquemment situé à la fin de l'item: si ces premières observations se confirmaient, elles laisseraient apparaître un modèle de production tendanciellement organisé par un ordre décondensation $\rightarrow$ condensation qui ferait du noyau une cible du geste énonciatif venant linguistiquement répondre au vouloir-dire initial. L'image du sillage que nous reprenions précédemment de Merleau-Ponty devrait alors se compléter de celle de la boule de neige: si le flot de mots proférés en réponse au vouloir- dire en 
est le sillage, la forme de ce sillage serait d'une certaine manière cumulative, la zone finale de la profération étant, assez logiquement d'ailleurs, un lieu propice à la synthèse de ce qui s'amorçait précédemment ${ }^{19}$. Différenciation sémiotique et syntagmatique d'unités que l'on peut toujours envisager dans des formats plus résorbés, l'item lexical l'est également dans la mesure où un parallèle peut être établi entre les types de valeurs sémantiques respectivement investies dans les prosodies sémantiques et les préférences sémantiques d'une part, et les phases de sens que Cadiot et Visetti ont dénommé motifs et profils dans leur modèle de la diversité lexicale d'autre part (Cadiot, Visetti, 2001): au titre des affinités entre motifs et prosodies, on peut en effet évoquer (outre la valeur « motivationnelle » que l'on a mentionné précédemment à propos des prosodies) l'investissement fréquent de valeurs sémantiques typiques (subjective, évaluative, modale, axiologique) ainsi que la difficulté rencontré à les dénommer de façon synthétique justifiant des gloses métalinguistiques par juxtaposition d'esquisses prenant souvent la forme de petits textes ${ }^{20}$; on reconnaîtra au contraire pour les préférences sémantiques et les profils le même type de logique paradigmatique (i.e. logique de classes) se laissant plus facilement approcher par une dénomination synthétique. En sorte que le dépliage sémiotique en quoi consiste un item lexical semble par ailleurs pouvoir s'homologuer avec un début de syntagmatisation des phases du sens évoquées, d'ailleurs plus faciles à percevoir ainsi que dans le format restreint d'un noyau où elles sont par définition plus indifférenciées.

\section{Résumons.}

En nous inspirant des analyses de la phénoménologie du langage, nous avons argumenté pour une extension de la problématique de la perception sémantique à celle d'une perception sémiotique, incluant donc la première, et qui ne s'identifierait pas à la «somme» ou à la seule corrélation de la perception des deux plans du signifié et du signifiant acquis indépendament, mais plutôt à une perception unitaire, au sein de laquelle le sens prévaut généralement dans le dispositif attentionnel, et où la netteté de l'opposition du signifiant et du signifié est a minima le corrélat d'une opération de thématisation dont la nature est très variable. Cette thématisation est alors, littéralement,

\footnotetext{
${ }^{19}$ On notera d'ailleurs que cet ordre allant d'éléments décumulés vers d'autres qui le sont moins est celui que l'on retrouve dans de nombreux énoncés oraux non planifiés, dans lesquels la périphérie gauche juxtapose des unités rythmiques monosémiques décumulant modalités, foyers énonciatif, cadre isotopique, etc. (cf notamment Missire, Rouayrenc, 2011), certes sans pour autant que la seconde partie de l'énoncé reprenne ensuite ces éléments de façon cumulé, ce qui serait donc le cas dans le cas de l'item lexical.

${ }^{20}$ Il faudrait en fait pour être complet mentionner également les affinités entre prosodie sémantique, isotopie et motif : entre prosodie sémantique et isotopie, il y a bien entendu cette vocation à la qualification syntagmatique large qui caractérise les fonds sémantiques. On a par ailleurs souligné la proximité entre isotopie et motif, en argumentant que les lexicalisations des isotopies dites «spécifiques» (que nous avons trouvé plus approprié de dénommer « hétérosystématiques ») justifiaient le recours à des lexèmes portant des motifs de façon à ce que la variation interne de l'isotopie en différents points de la chaîne syntagmatique puisse être «captée » par l'ouverture et l'indétermination foncière des motifs (cf. Missire, 2005, pp. 174-184).
} 
la condition d'une sémiogenèse, et cela revient à dire qu'il n'y a de signe que lorsque que l'activité énonciative/interprétative s'interrompt, et que le champ alors précipite localement en une unité : moins qu'une entité prenant place dans un champ qui lui serait comme une toile de fond, celle-ci se conçoit en effet davantage comme une conformation locale de ce champ, et c'est bien pour nous l'intérêt des travaux sur des grandeurs comparables aux items lexicaux que de pouvoir être interprétés dans ce sens, puisque l'identité de l'unité se confondra finalement avec ses coordonnées sur les différents axes de déploiement du champ, ceux-ci s'identifiant eux-mêmes aux catégories structurantes de l'analyse linguistique. Parce qu'il les articule dans sa méréologie interne, ce type d'unités apparaît ainsi comme pouvant inspirer un modèle sémiotique et perceptif des unités linguistiques moins simpliste que la traditionnelle dyade, modèle dont nous avons dans la deuxième section proposé quelques pistes d'élaboration à approfondir.

\section{Bibliographie}

BEDNAREK M. (2008), "Semantic preference and semantic prosody re-examined », dans Corpus linguistics and linguistic theory, 4-2, p. 119-139.

BOUQUET S. (2007), " Linguistique, interprétation et poésie, réponse à une "réclamation" d'Yves Bonnefoy », dans D. Lançon, P. Née (Eds), Yves Bonnefoy, poésie, savoirs et recherche, Paris : Hermann.

CADIOT P., VISETTI Y.-M. (2001), Pour une théorie des formes sémantiques, Motifs, Profils, Thèmes, Paris, PUF.

HUSSERL E. (1995), Leçons sur la théorie de la signification, Vrin, coll. Bibliothèques des textes philosophiques.

LOUW W. E. (1993), "Irony in the text or insincerity in the writer ? The diagnostic potential of semantic prosodies. » In M. Baker, G. Francis, E. Tognini-Bonelli (Eds.), Text and technology; in honour of John Sinclair, Amsterdam : John Benjamins.

LOUW W. E. (2000), « Contextual prosodic theory : bringing semantic prosodies to life ", In C. Heffer and H. Sauntson (Eds) Words in Context, A tribute to John Sinclair. On his Retirement, (p. 49-94), Birmingham : University of Birmingham. Réédité sur Texto ! Corpus et trucs, mis à jour le : 22/07/2008, URL : http://www.revuetexto.net/index.php?id=124.

MARTINET A. (1960/1980), Éléments de linguistique générale, Paris : Armand Colin.

MERLEAU-PONTY M. (1969), La prose du monde, Gallimard.

MISSIRE R. (2005), Sémantique des textes et modèle morphosémantique de l'interprétation, Thèse de l'Université de Toulouse II.

MISSIRE R. (2007), « Rythmes sémantiques et temporalité des parcours interprétatifs », dans M. Ballabriga, P. Mpondo-Dicka (Dir.), Rythme, Sens et textualités, Linguistique, 
sémiotique du discours, sémantique des textes, rhétorique, stylistique, poétique, Éditions Universitaires du Sud, p. 75-115.

MISSIRE R. (2010), «Unités linguistiques à signifiant discontinu, du morphème au texte - une approche néo-saussurienne », dans J.-P. Bronckart, C. Bota, E. Bulea (Eds.), Le projet de Ferdinand de Saussure, chap. 14, Genève, Droz, p. 289-312.

MISSIRE R., ROUAYRENC C (2013), «Sémantique du préambule : descriptions de la périphérie gauche de l'énoncé oral spontané », in R. Missire (dir.) Sémantique de l'oral spontané, Louvain, Academia Bruylant (à paraître).

PIOTROWSKI D. (2010), «Structuralisme et phénoménologie : le pont du signe », à paraître dans les Cahiers Ferdinand de Saussure.

RASTIER F. (1987), Sémantique interprétative, Paris, PUF. RASTIER F. (1991), Sémantique et recherche cognitive, Paris, PUF. RASTIER F. (2001), Arts et sciences du texte, Paris, PUF.

RASTIER F. (2003), «Formes sémantiques et textualité », dans D. Legallois (Ed.), Unité(s) du texte (p. 99-114), Cahiers du Crisco, 12, Université de Caen.

RASTIER F. (2007), «Passages ». Corpus, 6, Interprétation, contextes, codage.

ROSENTHAL V., VISETTI Y.-M. (1999), «Sens et temps de la Gestalt », Intellectica, 28, p. 147-227. ROSENTHAL V., VISETTI Y.-M. (2008), « Modèles et pensées de l'expression : perspectives microgénétiques », Intellectica, 50, p. 177-252.

SAUSSURE F. de (1972 [1916]), Cours de linguistique générale, Paris, Payot.

SAUSSURE F. de (2002), Écrits de linguistique générale, Paris, Gallimard.

SIEPMAN D. (2006), « Collocations et dictionnaires d'apprentissage onomasiologiques bilingues : questions aux théoriciens et pistes pour l'avenir » dans F. J. Hausmann, P. Blumenthal, Collocations, corpus, dictionnaires, Langue Française, juin 2006.

SINCLAIR J. (2004), Trust the text, language, corpus and discourse, London, New York : Routledge. VISETTI Y.-M., CADIOT P. (2006), Motifs et proverbes, essai de sémantique proverbiale, Paris, PUF.

WHITSITT S. (2005), "A critique of the concept of semantic prosody », in International journal of corpus linguistics, vol. 10, n³, p. 283-305.

\section{ANNEXES}

1. Coocurrences de Traiter de, friser, et frôler dans le corpus Le Monde (19912000). Source

Les voisins du Monde (http://www.irit.fr:8080/voisinsdelemonde/) 


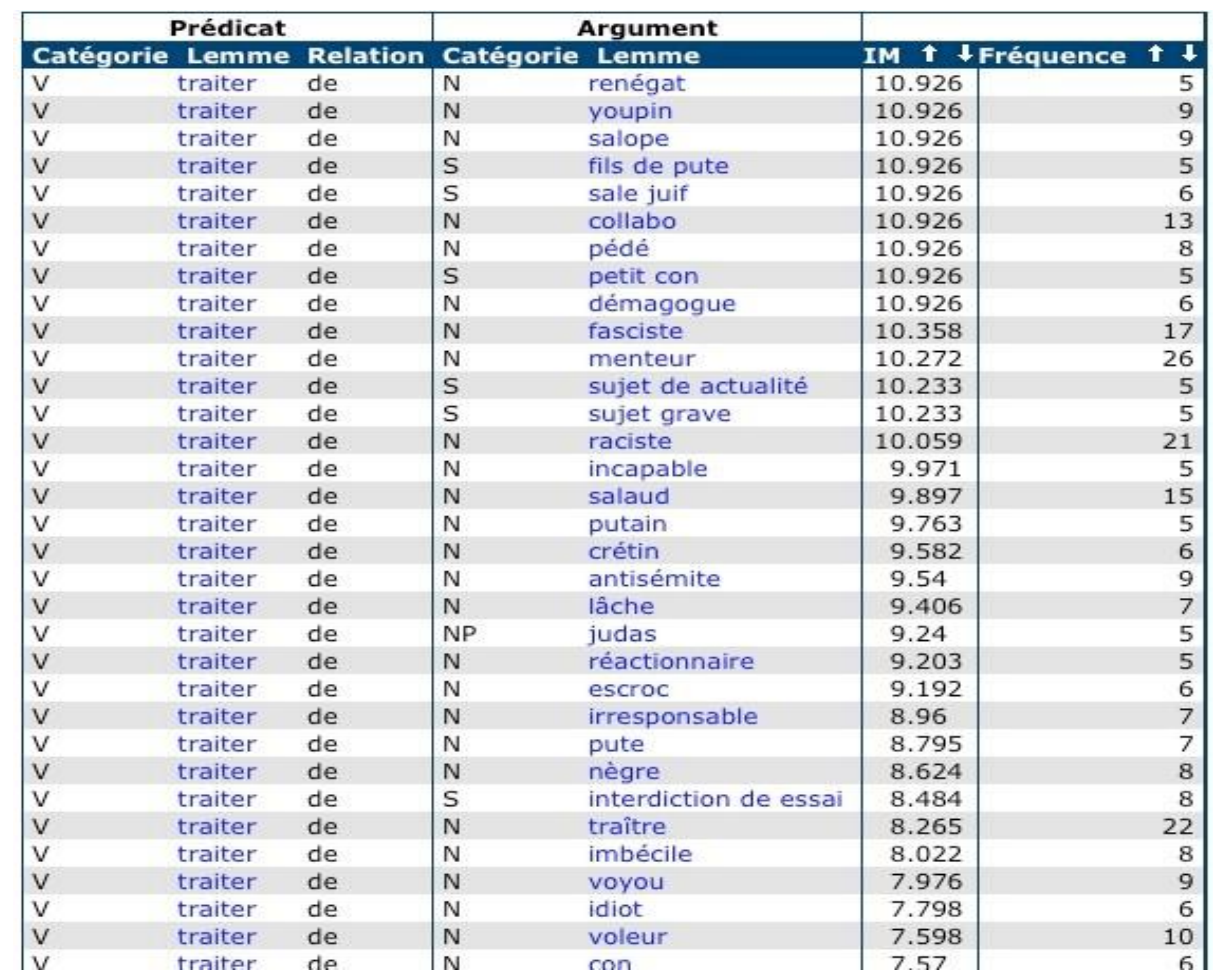

\begin{tabular}{|c|c|c|c|c|c|c|c|}
\hline \multicolumn{3}{|c|}{ Prédicat } & \multicolumn{2}{|c|}{\begin{tabular}{|c|} 
Argument \\
\end{tabular}} & \multirow{2}{*}{\multicolumn{2}{|c|}{ IM I \Fréquence }} & \multirow[b]{2}{*}{ 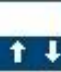 } \\
\hline Catégorie & Lemme & Relation & Catégorie & Lemme & & & \\
\hline $\mathrm{V}$ & frôler & obj & $\mathrm{N}$ & perversité & 10.572 & & 5 \\
\hline V & frôler & obj & $N$ & burlesque & 10.302 & & 6 \\
\hline V & frôler & obj & $\mathrm{N}$ & hystérie & 9.089 & & 8 \\
\hline V & frôler & obj & $N$ & ridicule & 9.024 & & 20 \\
\hline V & frôler & obj & $\mathrm{N}$ & caricature & 8.615 & & 14 \\
\hline V & frôler & obj & $N$ & perfection & 8.584 & & 14 \\
\hline V & frôler & obj & $N$ & délire & 8.498 & & 5 \\
\hline V & frôler & obj & $\mathrm{N}$ & absurde & 8.466 & & 6 \\
\hline V & frôler & obj & $N$ & abîme & 7.864 & & 5 \\
\hline V & frôler & obj & $N$ & barre & 7.487 & & 42 \\
\hline V & frôler & obj & $N$ & folie & 6.995 & & 11 \\
\hline V & frôler & obj & $N$ & désespoir & 6.896 & & 5 \\
\hline V & frôler & obj & $N$ & catastrophe & 6.781 & & 20 \\
\hline V & frôler & obj & $\mathrm{N}$ & cinquantaine & 6.674 & & 6 \\
\hline V & frôler & obj & $N$ & ennui & 6.626 & & 5 \\
\hline V & frôler & obj & $N$ & faillite & 6.242 & & 7 \\
\hline V & frôler & obj & $N$ & seuil & 6.231 & & 14 \\
\hline V & frôler & obj & N & exemplaire & 6.137 & & 16 \\
\hline V & frôler & obj & $N$ & record & 5.875 & & 11 \\
\hline V & frôler & obj & $N$ & incident & 5.422 & & 6 \\
\hline V & frôler & obj & $N$ & drame & 5.386 & & 6 \\
\hline V & frôler & obj & $N$ & limite & 5.259 & & 7 \\
\hline V & frôler & obj & $\mathrm{N}$ & mort & 4.922 & & 25 \\
\hline V & frôler & obj & $N$ & majorité & 4.915 & & 26 \\
\hline
\end{tabular}




\begin{tabular}{|c|c|c|c|c|c|c|c|}
\hline \multicolumn{3}{|c|}{ Prédicat } & \multicolumn{2}{|c|}{\begin{tabular}{|c|} 
Argument \\
\end{tabular}} & \multirow{2}{*}{\multicolumn{3}{|c|}{ IM † †Fréquence $\uparrow$}} \\
\hline Catégorie & Lemme & Relation & Catégorie & Lemme & & & \\
\hline V & friser & obj & $\mathrm{N}$ & indécence & 12.675 & & 17 \\
\hline V & friser & obj & $\mathrm{N}$ & académisme & 11.239 & & 5 \\
\hline V & friser & obj & $\mathrm{N}$ & insolence & 11.17 & & 8 \\
\hline V & friser & obj & N & inconscience & 10.277 & & 6 \\
\hline V & friser & obj & N & ridicule & 10.222 & & 34 \\
\hline V & friser & obj & N & paranoïa & 10.007 & & 5 \\
\hline V & friser & obj & N & hystérie & 9.875 & & 9 \\
\hline V & friser & obj & N & caricature & 9.477 & & 17 \\
\hline V & friser & obj & N & absurde & 9.421 & & 8 \\
\hline V & friser & obj & N & asphyxie & 9.048 & & 5 \\
\hline V & friser & obj & N & obsession & 8.278 & & 6 \\
\hline V & friser & obj & $\mathrm{N}$ & quarantaine & 7.471 & & 6 \\
\hline V & friser & obj & N & escroquerie & 7.224 & & 6 \\
\hline V & friser & obj & N & provocation & 7.193 & & 8 \\
\hline V & friser & $o b j$ & $\mathrm{~N}$ & catastrophe & 6.938 & & 12 \\
\hline V & friser & obj & N & cheveu & 6.883 & & 5 \\
\hline V & friser & $o b j$ & $\mathrm{~N}$ & racisme & 6.656 & & 5 \\
\hline V & friser & $o b j$ & N & milliard & 4.944 & & 30 \\
\hline V & friser & $o b j$ & $\mathrm{~N}$ & million & 4.432 & & 21 \\
\hline V & friser & $o b j$ & $\mathrm{~N}$ & majorité & 4.271 & & 7 \\
\hline
\end{tabular}

\section{Concordances de Parfois et toujours dans un corpus anonymisé de retranscription d'entretiens annuels d'évaluation d'agents de la fonction publique}

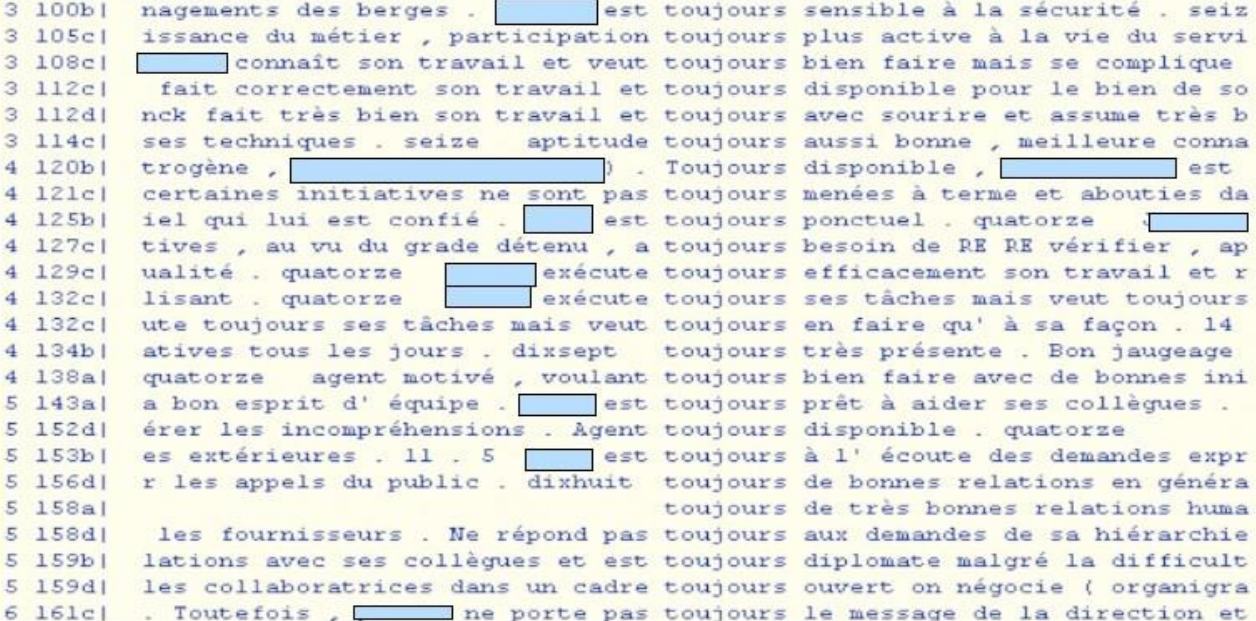

